\title{
Significance of cerebrospinal fluid inflammatory markers for diagnosing external ventricular drain-associated ventriculitis in patients with severe traumatic brain injury
}

\author{
Markus Lenski, MD, ${ }^{1}$ Annamaria Biczok, MD, ${ }^{1}$ Katrin Neufischer, ${ }^{1}$ Jörg-Christian Tonn, MD, ${ }^{1}$ \\ Josef Briegel, MD, ${ }^{2}$ and Niklas Thon, MD'
}

\begin{abstract}
${ }^{1}$ Neurochirurgische Klinik und Poliklinik, and ${ }^{2}$ Anästhesiologische Klinik, Klinikum Großhadern, Ludwig Maximilian Universität München, Munich, Germany
\end{abstract}

OBJECTIVE The aim of this study was to investigate the diagnostic potential of the inflammatory markers interleukin- 6 (IL-6), total leukocyte count (TLC), and protein in the CSF and IL-6, C-reactive protein, and white blood cell count in the serum for the early diagnosis of ventriculitis in patients with traumatic brain injury (TBI) and an external ventricular drain compared with patients without ventriculitis.

METHODS Retrospective data from 40 consecutive patients with TBI and an external ventricular drain treated in the authors' intensive care unit between 2013 and 2017 were analyzed. For all markers, arithmetical means and standard deviations, area under the curve (AUC), cutoff values, sensitivity, specificity, positive likelihood ratio (LR), and negative LR were calculated and correlated with presence or absence of ventriculitis.

RESULTS There were 35 patients without ventriculitis and 5 patients with ventriculitis. The mean \pm SD IL- 6 concentration in CSF was significantly increased, with $6519 \pm 4268 \mathrm{pg} / \mathrm{mL}$ at onset of ventriculitis compared with $1065 \pm 1705 \mathrm{pg} /$ $\mathrm{mL}$ in patients without ventriculitis $(p=0.04)$. Regarding inflammatory markers in CSF, IL-6 showed the highest diagnostic potential for differentiation between the presence and absence of ventriculitis (AUC 0.938, cutoff $4064 \mathrm{pg} / \mathrm{mL}$, sensitivity $100 \%$, specificity $92.3 \%$, positive LR 13 , and negative LR 0), followed by TLC (AUC 0.900 , cutoff $64.5 / \mu \mathrm{L}$, sensitivity $100 \%$, specificity $80 \%$, positive LR 5.0, and negative LR 0) and protein (AUC 0.876 , cutoff $31.5 \mathrm{mg} / \mathrm{dL}$, sensitivity $100 \%$, specificity $62.5 \%$, positive LR 2.7 , and negative LR 0 ).

CONCLUSIONS The level of IL-6 in CSF has the highest diagnostic value of all investigated inflammatory markers for detecting ventriculitis in TBI patients at an early stage. In particular, CSF IL-6 levels higher than the threshold of 4064 pg/ $\mathrm{mL}$ were significantly associated with the probability of ventriculitis.

https://thejns.org/doi/abs/10.3171/2019.8.FOCUS19407

KEYWORDS ventriculitis; external ventricular drain; cerebrospinal fluid; IL-6; traumatic brain injury; inflammatory marker; ventriculostomy

$\mathrm{S}$ INCE the initial primary tissue damage at the time of traumatic brain injury (TBI) is irreversible, further management focuses on early diagnosis and treatment of secondary damage and complications..$^{35}$ This secondary brain injury includes space-occupying intracranial hematoma, traumatic subarachnoid hemorrhage (SAH), cerebral edema, and hydrocephalus with critical impact on intracranial pressure (ICP). Approximately 57\% of patients with severe TBI need an external ventricular drain
$(\mathrm{EVD})^{8}$ for ICP control $^{29}$ or treatment of posttraumatic hydrocephalus. ${ }^{26,27}$

Ventriculitis is a frequent and dreaded complication of an external ventricular drain (EVD) device. ${ }^{33}$ It significantly increases morbidity and mortality in neurological ICU patients. ${ }^{1}$ In addition to secondary prophylaxis to prevent ventriculitis ${ }^{33}$ early diagnosis is extremely important..$^{14,15}$ While increasingly better criteria, including new inflammatory markers, have been established for the early

ABBREVIATIONS AUC = area under the curve; $\mathrm{CDC}=$ Centers for Disease Control and Prevention; $\mathrm{CRP}=\mathrm{C}$-reactive protein; EVD = external ventricular drain; $\mathrm{GCS}=$ Glasgow Coma Scale; ICP = intracranial pressure; IL-6 = interleukin-6; LR = likelihood ratio; ROC = receiver operating characteristic; SAH = subarachnoid hemorrhage; TBI $=$ traumatic brain injury; $T L C=$ total leukocyte count; $W B C C=$ white blood cell count.

SUBMITTED May 26, 2019. ACCEPTED August 23, 2019.

INCLUDE WHEN CITING DOI: 10.3171/2019.8.FOCUS19407. 
detection of ventriculitis after $\mathrm{SAH},{ }^{9}$ the diagnostic potential of inflammatory reactions in serum and CSF for the detection of ventriculitis after TBI has not yet been objectively assessed. Based on our experience with patients with $\mathrm{SAH},{ }^{14,15}$ we aimed to investigate whether the interleukin-6 (IL-6) level in CSF in particular may be useful for the early detection of bacterial ventriculitis after TBI and placement of an EVD.

\section{Methods}

This retrospective, single-center study includes all consecutive adult patients ( $>18$ years) with TBI and initial EVD implantation who were treated in our neurosurgical ICU between January 2013 and December 2017. Existence and severity of TBI was diagnosed on admission using Glasgow Coma Scale (GCS) scores and CT findings. ${ }^{29}$ The ethics review committee of the Ludwig Maximilian University of Munich approved this study.

Indications for EVD were invasive ICP monitoring in sedated and intubated patients or acute hydrocephalus observed on CT scanning. ${ }^{27}$ EVD implantation was performed under sterile conditions and antibiotic prophylaxis (1.5 g cefuroxime intravenously) in the emergency department under CT control or in the operating room. After the hair was shaved in the area of Kocher's point $(2.5 \mathrm{~cm}$ from the midline and approximately $12 \mathrm{~cm}$ posterior to the nasion but anterior to the coronal suture), the skin was disinfected with a povidone-iodine solution. ${ }^{15}$ The skin incision was made at Kocher's point, followed by a drill hole with a gimlet and catheter implantation into the lateral ventricle. A control scan confirmed correct positioning of the catheter and excluded a procedure-related hemorrhage. Subcutaneous tunneling was not routinely performed. A purse-string stitch sutured the wound and secured the EVD position. The wound and the exit point of the EVD were covered with sterile dressings, and the EVD was connected to a closed CSF collection system.

CSF was aspirated sterilely by a physician via a proximal 3-way stopcock. According to our standard operating procedures in TBI patients, the serum markers white blood cell count (WBCC), IL-6, and CRP and CSF markers IL-6, total leukocyte count (TLC), and protein were determined daily from the installation until the removal of the EVD. Serum and CSF markers were measured in the Department of Laboratory Medicine at our hospital. Measurements of biomarker levels were performed according to the manufacturer's instructions, and quality control was ensured. Blood and CSF specimens were not obtained solely for the purpose of this study.

EVD-associated ventriculitis in patients with TBI was defined in this study as culture-verified ventriculitis with a positive microbiological CSF culture, positive CSF Gram stain, or positive microbiological culture of the EVD tip.

The criteria for diagnosing meningitis and EVD-associated ventriculitis are based on the criteria for nosocomial infections from Centers for Disease Control and Prevention (CDC): 1) proof of an organism in the CSF in a microbiological testing method performed for purposes of clinical diagnosis and not for surveillance sampling; and 2) clinical worsening or new neurological symptoms together with altered laboratory CSF parameters (protein, glucose, and TLC). ${ }^{6,11,34}$ Most patients with severe TBI have a GCS score of 3 due to anesthesia and have altered CSF parameters due to the trauma. Thus, neurological and laboratory deterioration is difficult to detect and interpret and, according to current CDC criteria, unsuitable for diagnosing ventriculitis.

Microbiological testing was performed in TBI patients with suspected ventriculitis. Suspected ventriculitis was diagnosed concordantly by 2 experienced consultant doctors (a neurosurgeon and a neurointensive physician) in the field of neurointensive care. Physicians must be aware that contamination could lead to false-positive results. We tried to minimize the risk of contamination by strictly sterile aspiration of the CSF by a physician via the proximal 3-way stopcock. According to our standard operating procedures, 2-4 CSF tubes were collected for bacterial culture, Gram stain, and molecular testing. Furthermore, we prioritize multiple tests on small-volume samples $(<1$ $\mathrm{mL}$ ). The gold standard for diagnosing any type of infection is the proof of a pathogen in a microbiological testing method. As current CDC criteria for ventriculitis and meningitis are inadequate for TBI patients, we investigated only patients with culture-verified ventriculitis.

We used the inflammatory marker level at the time of first diagnosis of ventriculitis due to TBI (inflammatory marker level was measured on the same day when the specimen was obtained for microbiological testing) and compared it with levels in patients with TBI and an EVD without ventriculitis on the 12th day, as the mean time to infection was $11.6 \pm 2.7$ days. Normal distribution of data was investigated using the Kolmogorov-Smirnov test. We used receiver operating characteristic (ROC) curves with the corresponding area under the curve (AUC) to determine the diagnostic potential of inflammatory markers for predicting ventriculitis. The arithmetical mean \pm SD of biomarker levels in both groups were compared using the Student t-test. Mean values were considered to differ statistically significantly when $p<0.05$. Outcome parameters of this study were sensitivity, specificity, positive likelihood ratio (LR) and negative LR. Optimal thresholds were calculated using Youden's J statistic by maximizing sensitivity and specificity. Univariate analysis of risk factors was investigated using the chi-square test, and calculations were performed using SPSS (version 17, SPSS Inc.) and IBM SPSS (version 23.0, IBM Corp.) for Windows.

\section{Results}

Basic characteristics, including the injury pattern after head trauma, of the 40 patients are summarized in Table 1. In 17 patients (43\%), initial cranial CT revealed an open TBI (i.e., perforation of the scalp, fracture of the skull with or without rupture of the hard meninges, or CT scan with air inclusions situated in the extradural, subdural, or subarachnoid spaces or in the brain parenchyma ${ }^{3,5,31}$ ) with an indication for prophylactic antibiotic treatment with ceftriaxone. Thirty-five patients sustained a TBI and did not experience ventriculitis, and 5 patients developed ventriculitis. The detected pathogens are listed in Table 2. The mean time to infection was $11.6 \pm 2.7$ days $( \pm \mathrm{SD})$ after trauma. 
TABLE 1. Patient characteristics

\begin{tabular}{|c|c|}
\hline & No. of Patients (\%) \\
\hline Multiple trauma* & $18(45)$ \\
\hline |solated TBI & $22(55)$ \\
\hline Treatment & $40(100)$ \\
\hline Decompressive craniectomy & $13(33)$ \\
\hline Other neurosurgical procedure & $2(5)$ \\
\hline EVD \pm Codman Microsensor & $25(63)$ \\
\hline $\begin{array}{l}\text { Open head injury/acute traumatic pneuma- } \\
\text { tocephalus } †\end{array}$ & $17(43)$ \\
\hline Closed head injury & $23(57)$ \\
\hline \multicolumn{2}{|l|}{ Types of leading intracranial injury } \\
\hline $\mathrm{SDH}$ & $17(43)$ \\
\hline $\mathrm{EDH}$ & $3(8)$ \\
\hline $\mathrm{IPH}$ & $16(40)$ \\
\hline SAH & $4(10)$ \\
\hline Mechanism of injury & $40(100)$ \\
\hline Fall & $19(48)$ \\
\hline MVA & $4(10)$ \\
\hline MV/ped & $2(5)$ \\
\hline MCA & $3(8)$ \\
\hline Bicycle & $7(18)$ \\
\hline Suicidal head shot & $2(5)$ \\
\hline Fight & $1(3)$ \\
\hline Buried in construction debris & $2(5)$ \\
\hline Pupillomotor function & $40(100)$ \\
\hline Unilateral unreactive pupils & $11(28)$ \\
\hline Bilateral unreactive pupils & 7 (18) \\
\hline GCS score on admission & $40(100)$ \\
\hline 15 & $0(0)$ \\
\hline 14 & $0(0)$ \\
\hline 13 & $0(0)$ \\
\hline 12 & $0(0)$ \\
\hline 11 & $1(3)$ \\
\hline 10 & $3(8)$ \\
\hline 9 & $7(18)$ \\
\hline 8 & $3(8)$ \\
\hline 7 & $1(3)$ \\
\hline 6 & $1(3)$ \\
\hline 5 & $0(0)$ \\
\hline 4 & $0(0)$ \\
\hline 3 & $24(60)$ \\
\hline
\end{tabular}

$\mathrm{EDH}=$ epidural hematoma; IPH = intraparenchymal hemorrhage; $\mathrm{MCA}=$ motorcycle accident; $\mathrm{MVA}=$ motor vehicle accident; $\mathrm{MV} /$ ped = pedestrian struck by motor vehicle; $\mathrm{SDH}=$ subdural hematoma.

Patients received prophylactic antibiotic treatment with ceftriaxone for prevention of meningitis.

* Injury Severity Score $\geq 16$.

$\dagger$ Perforation of the scalp, fracture of the skull with or without rupture of the hard meninges, or CT scan with air inclusions situated in the extradural, subdural, subarachnoid spaces, or in the brain parenchyma.

Inflammatory marker levels were normally distributed. The mean CSF IL-6 levels were significantly increased in patients at onset of ventriculitis $(6519 \pm 4268 \mathrm{pg} / \mathrm{mL}$ in patients with ventriculitis vs $1065 \pm 1705 \mathrm{pg} / \mathrm{mL}$ in those without $[p=0.04])$. The AUC for the IL-6 level in CSF for predicting ventriculitis was 0.938 (Fig. 1). The opti-
TABLE 2. Isolated pathogens causing EVD-associated ventriculitis

\begin{tabular}{clll}
\hline $\begin{array}{c}\text { Case } \\
\text { No. }\end{array}$ & \multicolumn{1}{c}{ CSF } & Gram Stain & EVD Tip \\
\hline 1 & Staphylococcus epidermidis & & \\
\hline 2 & Escherichia coli & & \\
\hline 3 & S. epidermidis & S. epidermidis & S. epidermidis \\
\hline 4 & S. epidermidis & & \\
\hline 5 & Propionibacterium acnes & & \\
\hline
\end{tabular}

mal threshold was $4064 \mathrm{pg} / \mathrm{mL}$ with a sensitivity of $100 \%$, specificity of $92.3 \%$, positive LR of 13.0, and negative LR of 0 . The TLC level in CSF was significantly higher in patients with ventriculitis $(883 \pm 845 / \mu \mathrm{L}$ vs $13.5 \pm 16.0$ / $\mu \mathrm{L}$ in patients without ventriculitis). The corresponding AUC for the TLC level in CSF was 0.900 (cutoff 64.5/ $\mu \mathrm{L}$, sensitivity $100 \%$, specificity $80 \%$, positive LR 5.0, and negative LR 0). Results for serum WBCC, serum IL-6, serum CRP, and CSF protein are given in Table 3. The diagnostic significance of each of these parameters was lower than that of the IL-6 level in CSF. The respective ROC curves are depicted in Fig. 1. The predictive potentials of the IL-6 level in CSF at 24 hours and 48 hours before the positive microbiological culture are depicted in Fig. 2. Scatterplots of each biomarker are provided in Fig. 3. A univariate analysis of risk factors for predicting EVD-associated ventriculitis is given in Table 4. No risk factor reached statistical significance. Figure 4 depicts the number of patients with an EVD per day.

\section{Discussion}

Nearly 2.5 million people per year are affected by TBI in the United States..$^{13}$ Estimates predict that TBI will become the third most common cause of death and disability within the general population by $2020 . .^{12,13}$ Today, ICP monitoring is a safe and reliable method for ICP measurement with reported low complication rates (especially low infection rates). ${ }^{4}$ ICP monitoring is possible using an EVD or an ICP catheter. The indication for ICP monitoring is the lack of neurological assessment of a patient (Glasgow Coma Scale score 3-8) due to a TBI or drainage of acute hydrocephalus through an EVD. ${ }^{4}$ Since ICP monitoring has not been proven superior to imaging and clinical examination, the avoidance and early detection of deviceassociated infections is of central importance. ${ }^{4}$ This could prevent permanent device-associated secondary neurological deficits.

In line with previous TBI studies, the patients included in our study represent a typical adult patient cohort with severe TBI. Similar mechanisms of injury, types of injury, ventriculitis rates, and rates of patients with polytrauma have been reported previously. ${ }^{4,13,19,21}$ Nevertheless, based on the inclusion criteria of this study, our patients tended to have sustained more-severe TBI than patients in the STITCH[Trauma] trial ${ }^{19}$ the severity of TBI in our patients was similar to that of those in the BEST:TRIP trial. ${ }^{4}$ Approximately $60 \%$ of patients with severe TBI need 
Lenski et al.

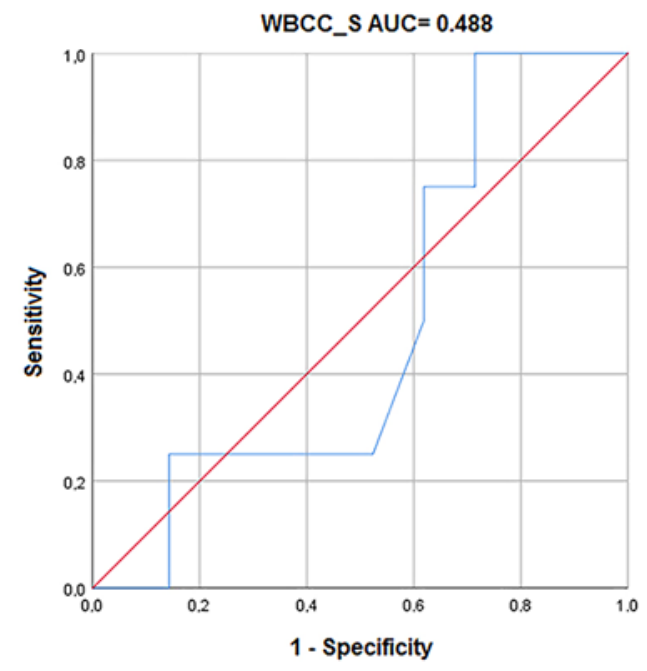

IL-6_S AUC $=0.635$

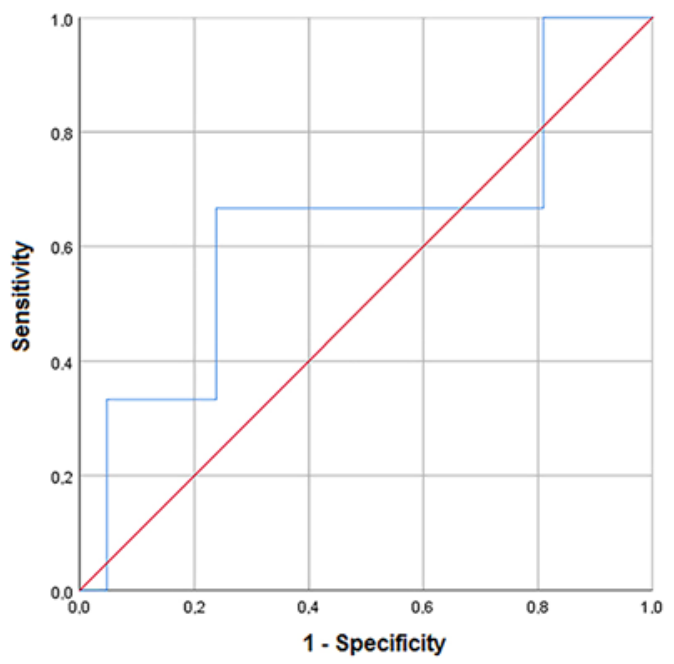

IL-6_CSF AUC $=0.938$

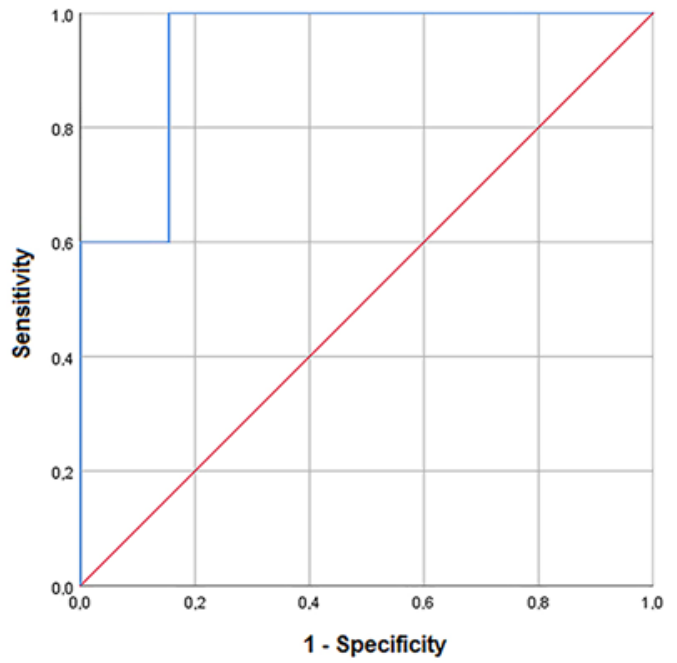

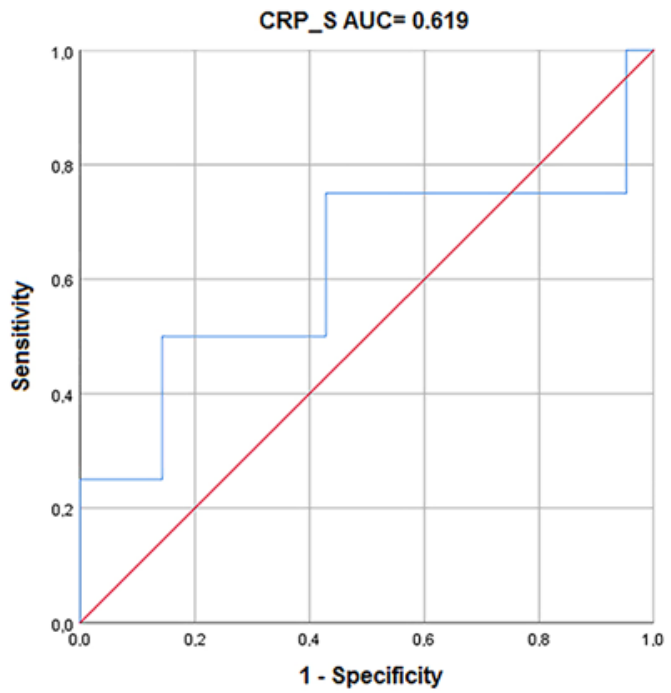

Protein_CSF AUC $=0.875$

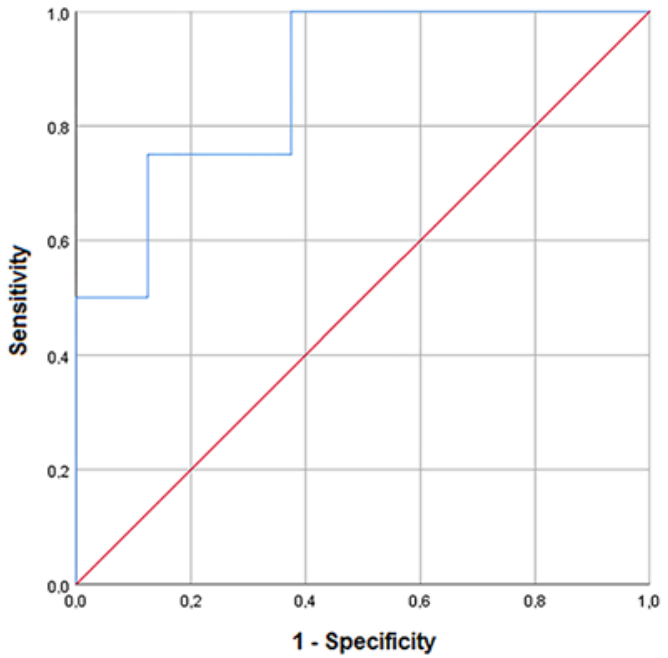

TLC_CSF AUC $=0.900$

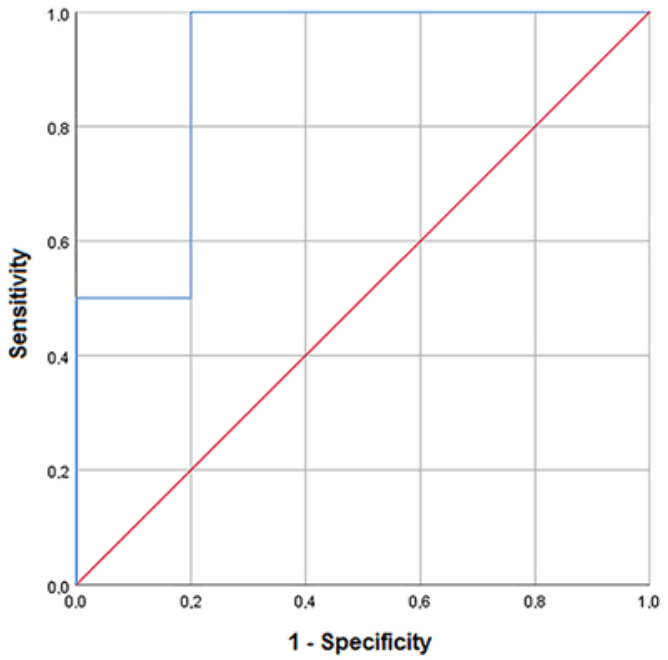

FIG. 1. ROC curves of serum and CSF markers for differentiating EVD-associated ventriculitis from an aseptic course. 


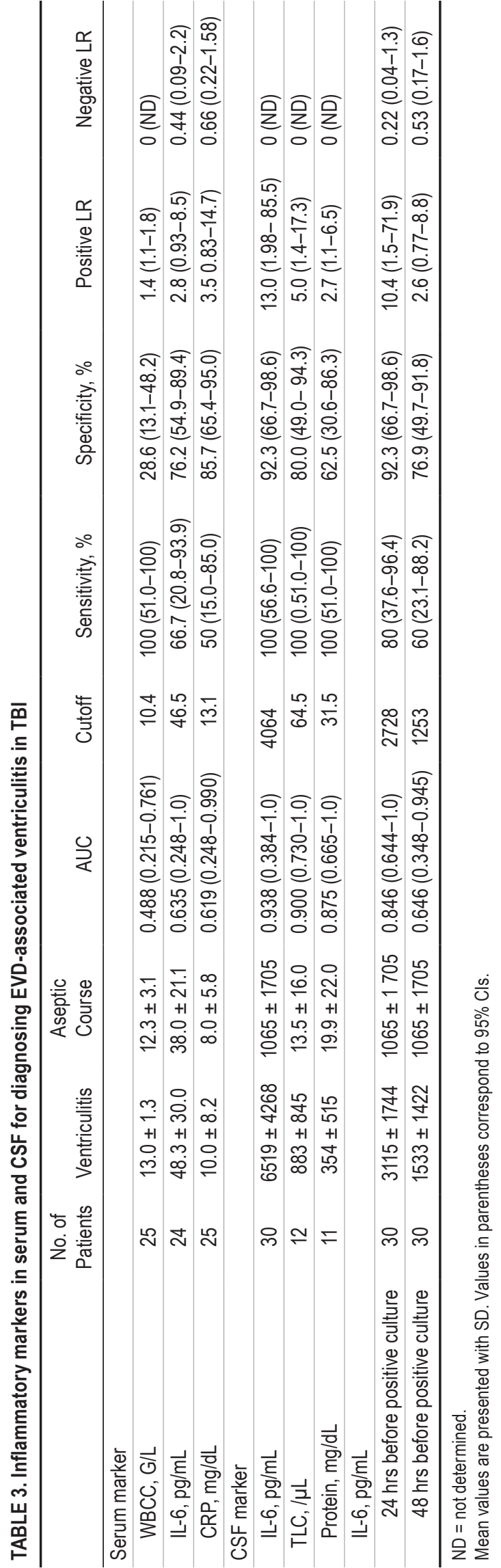

TABLE 4. Univariate analysis of risk factors for ventriculitis in patients with TBI and an EVD

\begin{tabular}{lccc}
\hline & $\begin{array}{c}\text { Ventriculitis } \\
\text { Group }\end{array}$ & $\begin{array}{c}\text { No Ventriculitis } \\
\text { Group }\end{array}$ & p Value \\
\hline No. of patients & 5 & 35 & \\
\hline Mean age \pm SD, yrs & $56 \pm 16$ & $57 \pm 21$ & 0.922 \\
\hline Sex (male/female) & $4 / 1$ & $29 / 6$ & 0.683 \\
\hline Antibiotics during ICU stay & 5 & 35 & 0.953 \\
\hline Secondary infarction & 1 & 2 & 0.298 \\
\hline Seizure & 1 & 2 & 0.386 \\
\hline Shunt dependency & 1 & 1 & 0.195 \\
\hline Immunosuppression & 0 & 0 & 0.877 \\
\hline Moderate TBI* & 2 & 9 & 0.716 \\
\hline Severe TBI† & 3 & 26 & 0.384 \\
\hline Sepsis & 1 & 6 & 0.567 \\
\hline Aspiration & 2 & 11 & 0.411 \\
\hline No comorbidities & 1 & 11 & 0.727 \\
\hline${ }^{*}$ GCS scores 9-12. & & & \\
† GCS scores 3-8. & & &
\end{tabular}

an EVD ${ }^{8}$ of these patients, between $1 \%$ and $30 \% 15,28,34$ develop EVD-associated ventriculitis. EVD-associated ventriculitis contributes significantly to the high morbidity and poor outcome of ICU patients..$^{14}$ Therefore, it is essential to detect and treat ventriculitis at an early stage. ${ }^{15}$ This may avoid ventriculitis-associated sequela, shorten hospital stay, and save costs for the healthcare system.

Currently, CSF culture remains the gold standard for diagnosing bacterial meningitis and is positive in $70 \%-$ $85 \%$ of cases prior to antibiotic administration., 2,17 However, patients with TBI and an EVD frequently receive periprocedural antibiotic prophylaxis, thereby reducing the diagnostic value of microbiological CSF samples. ${ }^{14,17}$ Moreover, in the early phase after severe TBI, patients are severely ill and need antibiotic treatment for various indications (e.g., infections of the lung, urinary tract infections, sepsis), which may further reduce the diagnostic value of microbiological CSF samples..$^{15}$ In addition, it takes at least 48 hours for routine CSF cultures to yield results, limiting their clinical use acutely. ${ }^{17}$

Here, we sought to identify the diagnostic potential of routine inflammatory markers in CSF and serum for early detection of ventriculitis after TBI and EVD implantation. We presented ROC curves and thresholds of common serum and CSF biomarkers on the day of onset of EVD-associated ventriculitis compared with noninfectious controls, which can support clinical decisionmaking. We identified IL-6 in the CSF to be a diagnostic marker with high diagnostic potential and a cutoff value of $4064 \mathrm{pg} / \mathrm{mL}$ for diagnosing EVD-associated ventriculitis in TBI patients. The respective values for IL-6 in serum did not reach similar diagnostic power. Moreover, IL-6 in CSF was a useful early predictive marker 24 hours before EVD-associated ventriculitis became manifest.

To the best of our knowledge, the diagnostic power of IL-6 for early diagnosis of EVD-associated ventriculitis after TBI has not been defined for clinical routine use so 


\section{IL-6_CSF 24h before positive culture $A U C=0.846$}

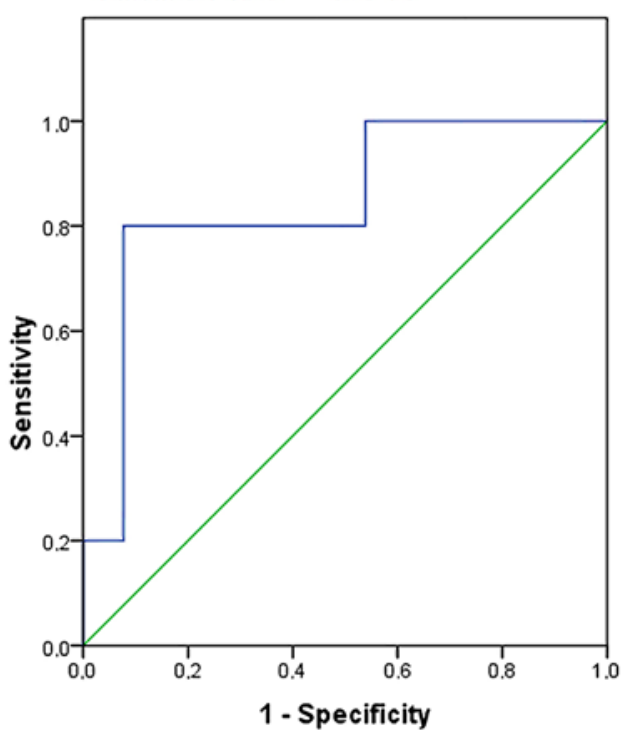

IL-6_CSF 48 h before positive culture AUC $=0.646$

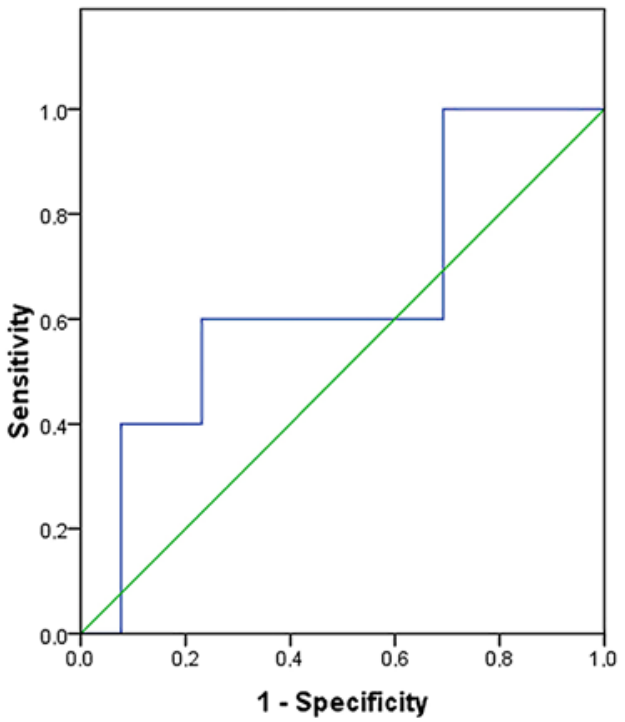

FIG. 2. ROC curves of the predictive potential of CSF IL-6 24 hours and 48 hours before positive culture.

far. ${ }^{14}$ However, its diagnostic significance can be derived from other neurological diseases.

One study investigated the role of IL-6 in CSF for predicting postoperative EVD-associated ventriculitis after neurosurgical procedures. ${ }^{17}$ IL-6 in CSF was significantly increased in patients with ventriculitis, and it had a moderate diagnostic potential for diagnosing ventriculitis on the day of fever rise. The calculated optimal cutoff value was similar to ours. ${ }^{17}$ Moreover, the usefulness of IL-6 in CSF for diagnosing ventriculitis has been shown in several studies of patients with aneurysmal SAH. ${ }^{10,14}$ Again, the diagnostic potential of IL-6 in CSF and respective cutoff values were in line with our results. ${ }^{4}$ It has been concluded that IL-6 in CSF after SAH could be an early marker for predicting ventriculitis. ${ }^{10}$ However, conflicting results have been shown regarding the usefulness of IL-6 in CSF for diagnosing bacterial meningitis in children ${ }^{25}$ and adults. ${ }^{24,32}$

In addition to its diagnostic potential for early diagnosis of ventriculitis, IL-6 in CSF has also been investigated regarding its association with injury severity and neurological outcome. Initially, a neuroprotective effect by elevated IL- 6 concentrations in CSF after TBI with improved clinical outcome was assumed. ${ }^{30}$ Two more recent studies show, however, that persistently elevated IL-6 concentrations in CSF correlate with injury severity and increase the odds for unfavorable global outcome..$^{13,21}$ In particular, IL-6 concentrations higher than $2000 \mathrm{pg} / \mathrm{mL}$ in CSF have a direct prognostic significance for predicting worse neurological outcome after TBI. ${ }^{13,21}$ This threshold is about half as high as the cutoff value for predicting ventriculitis after TBI. Vasospasm can also potentially occur in the context of TBI. Vasospasm has been shown to increase CSF IL-6 levels in SAH patients. ${ }^{14}$ This must also be considered in the assessment of CSF IL-6 increases in TBI patients.

The role of routinely determined biomarkers in the CSF such as TLC, percentage of neutrophils, glucose, and protein for diagnosing ventriculitis after TBI is unclear. ${ }^{15}$ While many biomarker studies did not include patients with TBI, ${ }^{10,14,15,23}$ our literature search identified 3 main studies that included subpopulations of patients with TBI and examined CSF inflammatory markers for diagnosing ventriculitis. ${ }^{17,18,34}$ In our study, TLC in CSF was significantly increased in patients with ventriculitis and had a very good diagnostic potential for predicting EVD-associated ventriculitis (cutoff value $64.5 / \mu \mathrm{L}$ ). This finding is in line with those of previous reports. ${ }^{7,15}$ Two studies confirmed significantly increased TLC in patients with ventriculitis, but results regarding glucose, protein, and percentage of neutrophils in CSF or serum markers WBCC and CRP were not conclusive. ${ }^{17,34}$ Another recently published study showed that the cell index (ratio of leukocytes to erythrocytes in CSF and leukocytes to erythrocytes in the peripheral blood) had a good diagnostic potential for predicting ventriculitis. ${ }^{18,23}$ Since the TLC in CSF already showed good diagnostic potential for diagnosing ventriculitis in this and other studies of TBI patients, it remains unclear whether the determination of the cell index could provide further diagnostic information.

Two studies about the diagnostic potential of biomarkers for diagnosing ventriculitis in patients with $\mathrm{SAH}$ reported a good diagnostic potential of TLC in $\mathrm{CSF}^{7,15}$ (cutoff value $635 / \mu \mathrm{L}$ ), while one study with a consecutive cohort of patients with EVD detected no significant difference in the mean concentrations of TLC in CSF. ${ }^{28}$ The clinical value of protein in CSF for predicting ventriculitis is also unclear. One study reported moderate diagnostic potential for protein in $\mathrm{CSF}^{14}$ and another reported no significant difference of mean protein concentrations in patients with or without ventriculitis. ${ }^{28}$ The percentage of neutrophils in CSF was a useful marker for predicting ventriculitis in 2 previous studies. ${ }^{7,14}$ Insufficient diagnostic potential has been described for serum IL-6 and WBCC.,14,15 For serum 

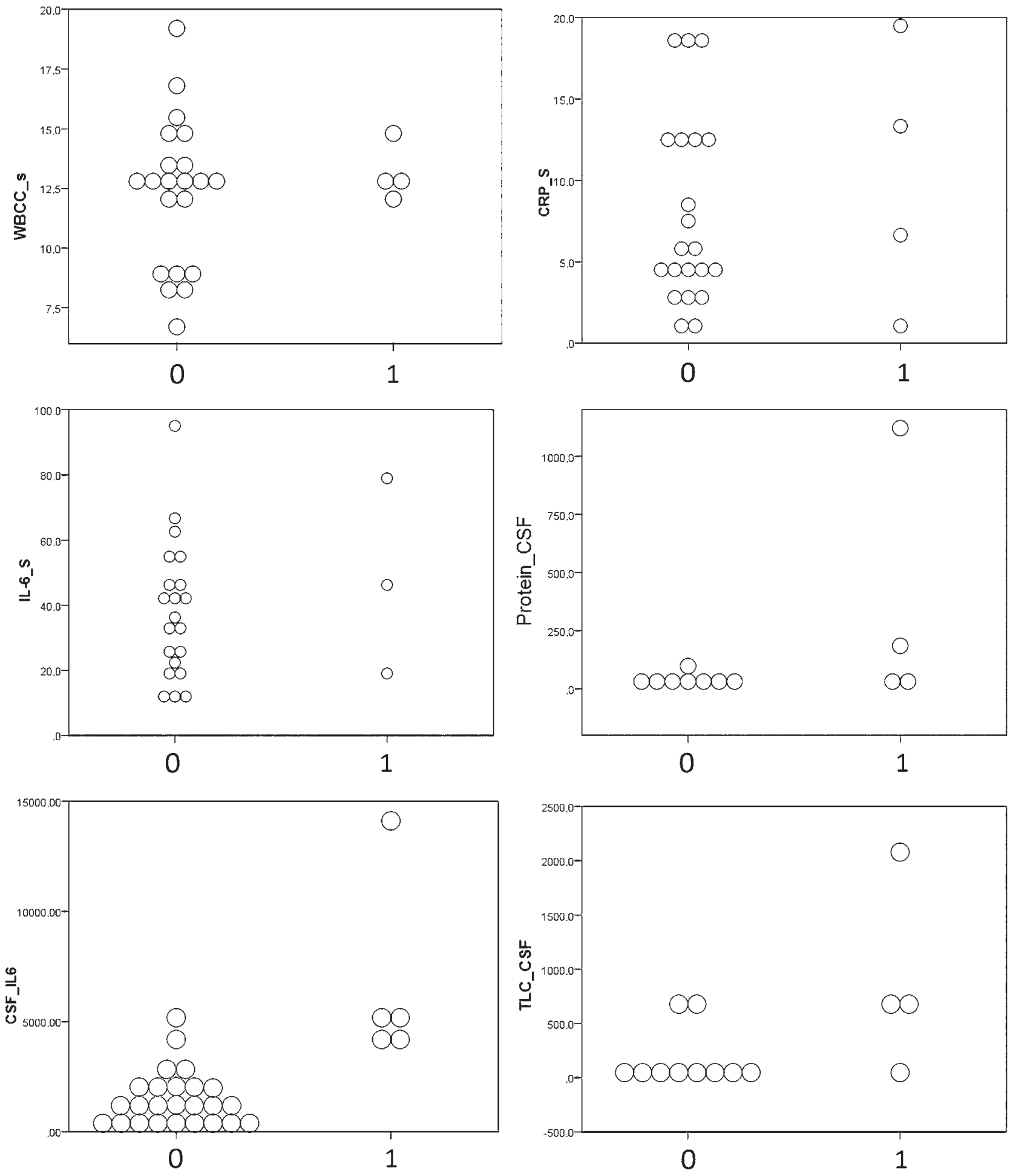

FIG. 3. Scatterplots of serum and CSF inflammatory marker levels in infectious and aseptic patients.

CRP, the study situation is unclear. Good and insufficient diagnostic potential have been described so far. ${ }^{7,14,15,34}$ It is concluded that cutoff values of TLC in CSF for diagnosing ventriculitis differ widely in TBI and SAH patients, but the role of protein in CSF, percentage of neutrophils in CSF, serum CRP, and serum WBCC remains unclear in patients with TBI or SAH and deserves further investigation.
The strength of this study is the strict criteria for ventriculitis after TBI and the homogeneous study population of patients with TBI. Other studies have been limited by a heterogeneous study population of patients who experienced numerous types of brain injury (e.g., SAH, intracranial bleeding, intraventricular bleeding, craniotomy, EVD only). ${ }^{7,17,18,23,28,34}$ Limitations include that our study 


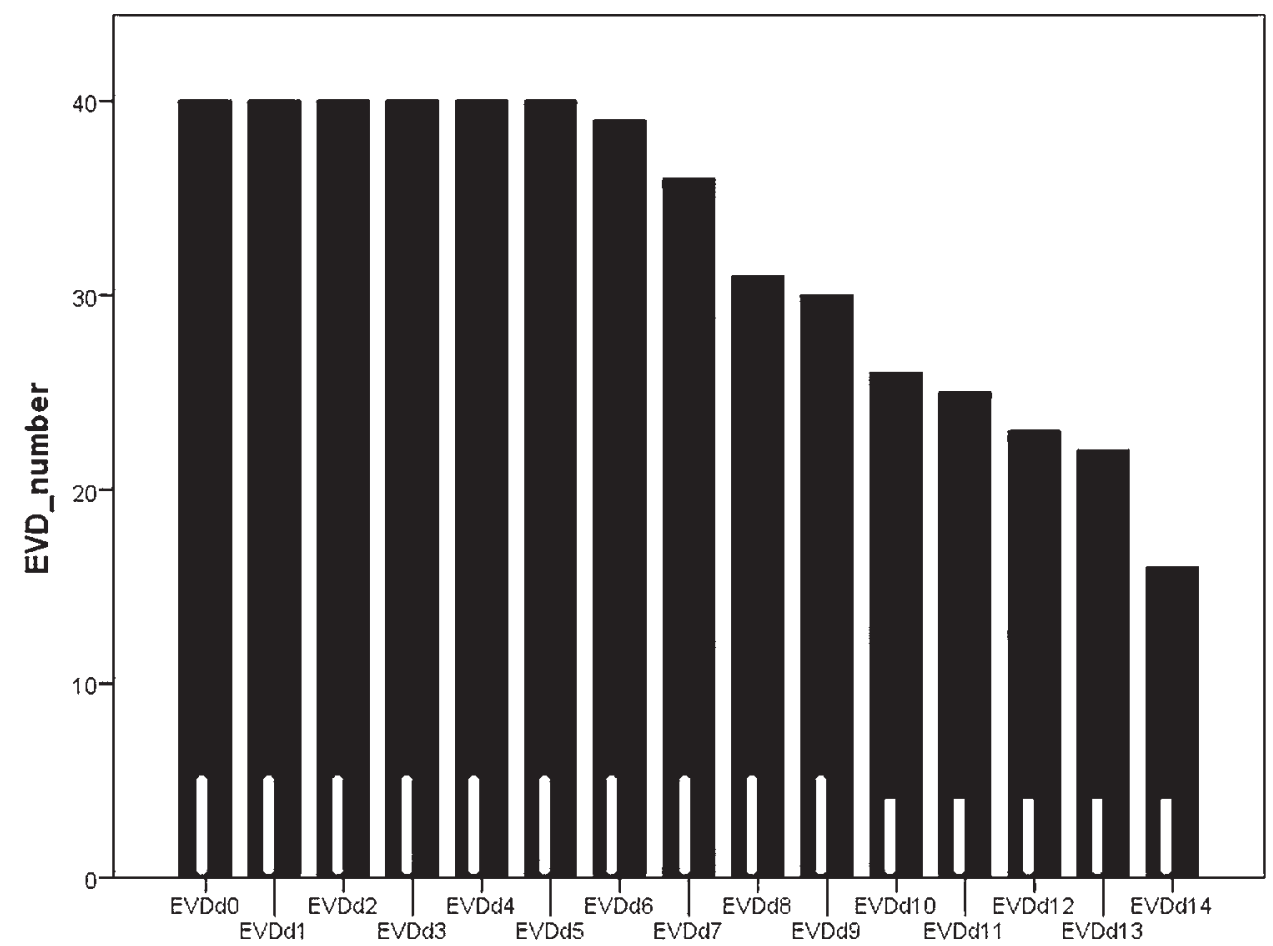

FIG. 4. Number of patients with EVDs per day after traumatic brain injury.

was designed as a retrospective clinical study. Therefore, data acquisition may not have been as accurate as that in prospective clinical studies. A high percentage of TBI patients had an acute traumatic pneumocephalus. These patients received prophylactic antibiotic treatment with ceftriaxone. Furthermore, all patients underwent perioperative antibiotic prophylaxis with cefuroxime prior to EVD implantation. Antibiotic treatment lowers the sensitivity of microbiological testing, ${ }^{16}$ especially in patients with ventriculitis. ${ }^{14}$ Patients with culture-negative ventriculitis may have been misclassified as not having bacterial ventriculitis in this study. This is a common problem of inflammatory marker studies..$^{15}$ In addition, the contamination of microbiological samples is a common problem in clinical routine. Despite all the precautions described in Methods, contamination can never be safely excluded. ${ }^{20}$ However, we think that we have captured the inflammatory marker levels in a representative and exactly defined study population that was treated by a strictly standardized operating procedure.

\section{Conclusions}

Diagnosing EVD-associated ventriculitis in patients with TBI at an early stage is challenging. Daily supervision of clinical symptoms in sedated patients and daily determination of biomarker levels in the CSF may be essential. IL-6 in CSF is significantly increased after TBI in patients with ventriculitis. Patients with a CSF IL-6 level greater than $4064 \mathrm{pg} / \mathrm{mL}$ have a drastically increased posttest probability for ventriculitis. Future prospective studies will show whether additional inflammatory markers in the CSF can further increase the diagnostic accuracy of current inflammatory markers.

\section{References}

1. Beer R, Lackner P, Pfausler B, Schmutzhard E: Nosocomial ventriculitis and meningitis in neurocritical care patients. J Neurol 255:1617-1624, 2008

2. Bohr V, Paulson OB, Rasmussen N: Pneumococcal meningitis. Late neurologic sequelae and features of prognostic impact. Arch Neurol 41:1045-1049, 1984

3. Chen L, Bao Y, Liang Y, Wang Y, Jiang J: Surgical management and outcomes of non-missile open head injury: Report of 44 cases from a single trauma centre. Brain Inj 30:318323, 2016

4. Chesnut RM, Temkin N, Carney N, Dikmen S, Rondina C, Videtta W, et al: A trial of intracranial-pressure monitoring in traumatic brain injury. N Engl J Med 367:2471-2481, 2012

5. Eftekhar B, Ghodsi M, Hadadi A, Taghipoor M, Sigarchi SZ, Rahimi-Movaghar V, et al: Prophylactic antibiotic for prevention of posttraumatic meningitis after traumatic pneumocephalus: design and rationale of a placebo-controlled randomized multicenter trial [ISRCTN71132784]. Trials 7:2, 2006

6. Garner JS, Jarvis WR, Emori TG, Horan TC, Hughes JM: CDC definitions for nosocomial infections, 1988. Am J Infect Control 16:128-140, 1988

7. Gordon M, Ramirez P, Soriano A, Palomo M, Lopez-Ferraz C, Villarreal E, et al: Diagnosing external ventricular drain-related ventriculitis by means of local inflammatory response: soluble triggering receptor expressed on myeloid cells-1. Crit Care 18:567, 2014

8. Griesdale DE, McEwen J, Kurth T, Chittock DR: External ventricular drains and mortality in patients with severe traumatic brain injury. Can J Neurol Sci 37:43-48, 2010

9. Hoogmoed J, van de Beek D, Coert BA, Horn J, Vandertop WP, Verbaan D: Clinical and laboratory characteristics for the diagnosis of bacterial ventriculitis after aneurysmal subarachnoid hemorrhage. Neurocrit Care 26:362-370, 2017

10. Hopkins SJ, McMahon CJ, Singh N, Galea J, Hoadley M, 
Scarth S, et al: Cerebrospinal fluid and plasma cytokines after subarachnoid haemorrhage: CSF interleukin-6 may be an early marker of infection. J Neuroinflammation 9:255-263, 2012

11. Horan TC, Andrus M, Dudeck MA: CDC/NHSN surveillance definition of health care-associated infection and criteria for specific types of infections in the acute care setting. Am J Infect Control 36:309-332, 2008

12. Hyder AA, Wunderlich CA, Puvanachandra P, Gururaj G, Kobusingye OC: The impact of traumatic brain injuries: a global perspective. NeuroRehabilitation 22:341-353, 2007

13. Kumar RG, Diamond ML, Boles JA, Berger RP, Tisherman SA, Kochanek PM, et al: Acute CSF interleukin-6 trajectories after TBI: associations with neuroinflammation, polytrauma, and outcome. Brain Behav Immun 45:253-262, 2015

14. Lenski M, Huge V, Briegel J, Tonn JC, Schichor C, Thon N: Interleukin 6 in the cerebrospinal fluid as a biomarker for onset of vasospasm and ventriculitis after severe subarachnoid hemorrhage. World Neurosurg 99:132-139, 2017

15. Lenski M, Huge V, Schmutzer M, Ueberschaer M, Briegel J, Tonn JC, et al: Inflammatory markers in serum and cerebrospinal fluid for early detection of external ventricular drainassociated ventriculitis in patients with subarachnoid hemorrhage. J Neurosurg Anesthesiol 31:227-233, 2019

16. Lenski M, Scherer MA: Synovial IL-6 as inflammatory marker in periprosthetic joint infections. J Arthroplasty 29:1105-1109, 2014

17. Liu ZH, Tu PH, Chen NY, Yip PK, Bowes AL, Lee CC, et al: Raised proinflammatory cytokine production within cerebrospinal fluid precedes fever onset in patients with neurosurgery-associated bacterial meningitis. Crit Care Med 43:2416-2428, 2015

18. Lunardi LW, Zimmer ER, Dos Santos SC, Merzoni J, Portela LV, Stefani MA: Cell index in the diagnosis of external ventricular drain-related infections. World Neurosurg 106:504508, 2017

19. Mendelow AD, Gregson BA, Rowan EN, Francis R, McColl E, McNamee P, et al: Early surgery versus initial conservative treatment in patients with traumatic intracerebral hemorrhage (STITCH[Trauma]): the first randomized trial. J Neurotrauma 32:1312-1323, 2015

20. Miller JM, Binnicker MJ, Campbell S, Carroll KC, Chapin $\mathrm{KC}$, Gilligan $\mathrm{PH}$, et al: A guide to utilization of the microbiology laboratory for diagnosis of infectious diseases: 2018 update by the Infectious Diseases Society of America and the American Society for Microbiology. Clin Infect Dis 67:813816, 2018

21. Nwachuku EL, Puccio AM, Adeboye A, Chang YF, Kim J, Okonkwo DO: Time course of cerebrospinal fluid inflammatory biomarkers and relationship to 6-month neurologic outcome in adult severe traumatic brain injury. Clin Neurol Neurosurg 149:1-5, 2016

22. Parikh S, Koch M, Narayan RK: Traumatic brain injury. Int Anesthesiol Clin 45:119-135, 2007

23. Pfausler B, Beer R, Engelhardt K, Kemmler G, Mohsenipour I, Schmutzhard E: Cell index-a new parameter for the early diagnosis of ventriculostomy (external ventricular drainage)related ventriculitis in patients with intraventricular hemorrhage? Acta Neurochir (Wien) 146:477-481, 2004

24. Pinto Junior VL, Rebelo MC, Gomes RN, Assis EF, CastroFaria-Neto HC, Bóia MN: IL-6 and IL-8 in cerebrospinal fluid from patients with aseptic meningitis and bacterial meningitis: their potential role as a marker for differential diagnosis. Braz J Infect Dis 15:156-158, 2011
25. Prasad R, Kapoor R, Srivastava R, Mishra OP, Singh TB: Cerebrospinal fluid TNF- $\alpha$, IL-6, and IL-8 in children with bacterial meningitis. Pediatr Neurol 50:60-65, 2014

26. Rumalla K, Letchuman V, Smith KA, Arnold PM: Hydrocephalus in pediatric traumatic brain injury: national incidence, risk factors, and outcomes in 124,444 hospitalized patients. Pediatr Neurol 80:70-76, 2018

27. Sakai H, Takagi H, Okada K, Tanabe T, Morii S, Ohwada T, et al: [Acute traumatic hydrocephalus.] No Shinkei Geka 12:205-209, 1984 (Japanese)

28. Schade RP, Schinkel J, Roelandse FW, Geskus RB, Visser LG, van Dijk JM, et al: Lack of value of routine analysis of cerebrospinal fluid for prediction and diagnosis of external drainage-related bacterial meningitis. J Neurosurg 104:101108, 2006

29. Seule M, Brunner T, Mack A, Hildebrandt G, Fournier JY: Neurosurgical and intensive care management of traumatic brain injury. Facial Plast Surg 31:325-331, 2015

30. Singhal A, Baker AJ, Hare GM, Reinders FX, Schlichter LC, Moulton RJ: Association between cerebrospinal fluid interleukin- 6 concentrations and outcome after severe human traumatic brain injury. J Neurotrauma 19:929-937, 2002

31. Steudel WI, Hacker H: Prognosis, incidence and management of acute traumatic intracranial pneumocephalus. A retrospective analysis of 49 cases. Acta Neurochir (Wien) 80:93-99, 1986

32. Takahashi W, Nakada TA, Abe R, Tanaka K, Matsumura Y, Oda S: Usefulness of interleukin 6 levels in the cerebrospinal fluid for the diagnosis of bacterial meningitis. J Crit Care 29:693.e1-693.e6, 2014

33. Tavakoli S, Peitz G, Ares W, Hafeez S, Grandhi R: Complications of invasive intracranial pressure monitoring devices in neurocritical care. Neurosurg Focus 43(5):E6, 2017

34. Walti LN, Conen A, Coward J, Jost GF, Trampuz A: Characteristics of infections associated with external ventricular drains of cerebrospinal fluid. J Infect 66:424-431, 2013

35. Xiong Y, Gu Q, Peterson PL, Muizelaar JP, Lee CP: Mitochondrial dysfunction and calcium perturbation induced by traumatic brain injury. J Neurotrauma 14:23-34, 1997

\section{Disclosures}

The authors report no conflict of interest concerning the materials or methods used in this study or the findings specified in this paper.

\section{Author Contributions}

Conception and design: Lenski, Biczok, Tonn, Briegel, Thon. Acquisition of data: Lenski, Biczok, Neufischer, Briegel. Analysis and interpretation of data: Lenski, Briegel, Thon. Drafting the article: Lenski, Tonn, Briegel, Thon. Critically revising the article: Lenski, Tonn, Briegel, Thon. Reviewed submitted version of manuscript: Lenski, Tonn, Briegel, Thon. Approved the final version of the manuscript on behalf of all authors: Lenski. Statistical analysis: Lenski, Tonn, Briegel, Thon. Administrative/technical/ material support: Briegel, Thon. Study supervision: Thon.

\section{Correspondence}

Markus Lenski: Neurochirurgische Klinik und Poliklinik, Klinikum der Universität München, Munich, Germany. markus. lenski@med.uni-muenchen.de. 